\title{
Cathodoluminescence system for a scanning electron microscope using an optical fiber for light collection
}

\author{
Michael E. Hoenk and Kerry J. Vahala \\ Department of Applied Physics, 128-95, California Institute of Technology, Pasadena, California 91125 \\ (Received 20 May 1988; accepted for publication 20 October 1988)

\begin{abstract}
We describe a novel hight collection system for cathodoluminescence scanning electron microscopy. Cathodoluminescence emitted from the sample surface enters directly into the facet of an optical fiber, which is held less than a millimeter away from the sample to optimize the collection efficiency. The fiber is small enough that it has a minimal effect on access to the sample by other detection apparatus. Three axis positioning of the fiber is accomplished with motorized translation stages located in the sample chamber. Techniques for generating cathodoluminescence images and local spectra are discussed. The techniques are applied to oval defects in molecular beam epitaxially grown GaAs/AlGaAs epilayers, and the results are presented.
\end{abstract}

\section{INTRODUCTION}

Cathodoluminescence $(\mathrm{CL})$ is the emission of light from a material as a result of excitation by an elcctron beam. In conjunction with the imaging capabilities of a scanning electron microscope (SEM), it is a powerful tool for the study and characterization of small features in direct gap semiconductors.'

A scanning electron microscope generates a beam of electrons which is focused to a small spot on the sample surface (Fig. 1). The beam is swept across an area of the sample in a raster pattern. In the conventional mode of operation, the image is formed by detecting the emission of secondary electrons from the sample surface, and electronically correlating the secondary electron signal with the position of the beam. Other signals, carrying different information about the sample, can also be used for image formation. For

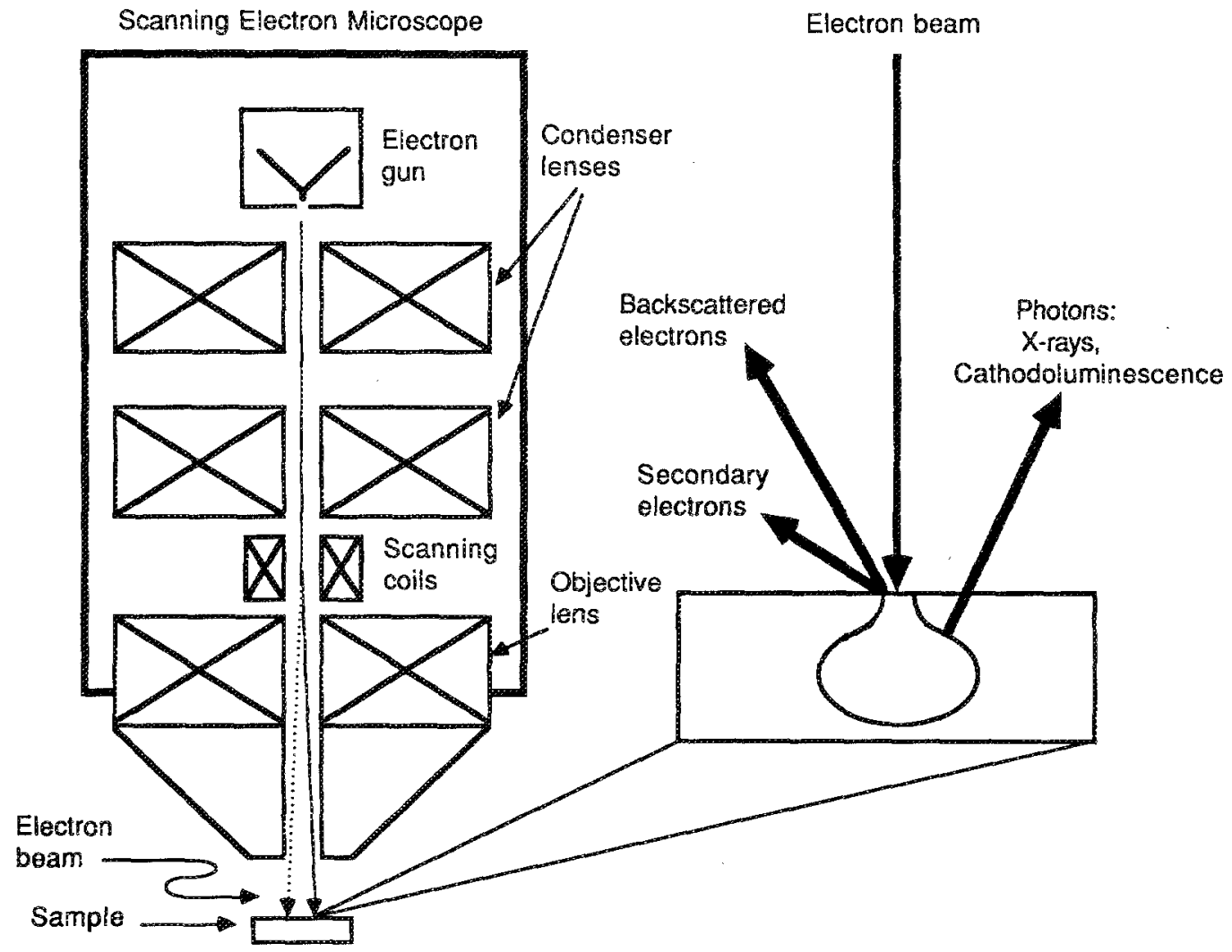

Fro. 1. Schematic diagram of clectron beam excitation of a samplc. The scanning electron microscope column is illustrated on the lcf, with the electron beam originating at the fllament and passing through the two condertser lenses, the scaming coils, and the objective lens, finally impinging on the sample as a fine probe. On the right, the interaction of the electron beam with the sample is illustrated, showing the interaction volume of the beam with the sample, and some of the products of the interaction. 
example, backscattered electrons can be collected with a special detector to produce an image which is sensitive to variations in the material composition of the surface.

The electron beam induces a variety of excitations that result in emitted radiation. In particular, electron-hole pairs are produced in the interaction volume of the electron beam with the sample. The carriers generated by this process exist for a characteristic period of time, during which they undergo intraband relaxation and diffusion/drift before recombining. Recombination can be accompanied by the emission of a photon, which is particularly likely if the sample is a direct gap semiconductor. In this case, the photon will have an energy near the local band gap energy of the semiconductor. The light generated by this process (cathodoluminescence) can be used to study the local properties of the material, and the results can be correlated with information from other SEM imaging techniques.

In this paper we report the development of a novel CL detection system which uses an optical fiber for direct collection of light from the surface of the sample. We discuss techniques for generating spectrally resolved cathodoluminescence (SRCL) images and local CL spectra, and present results of a study of oval defects in GaAs/AlGaAs epilayers grown by molecular beam epitaxy $(\mathrm{MBE}) .^{2}$

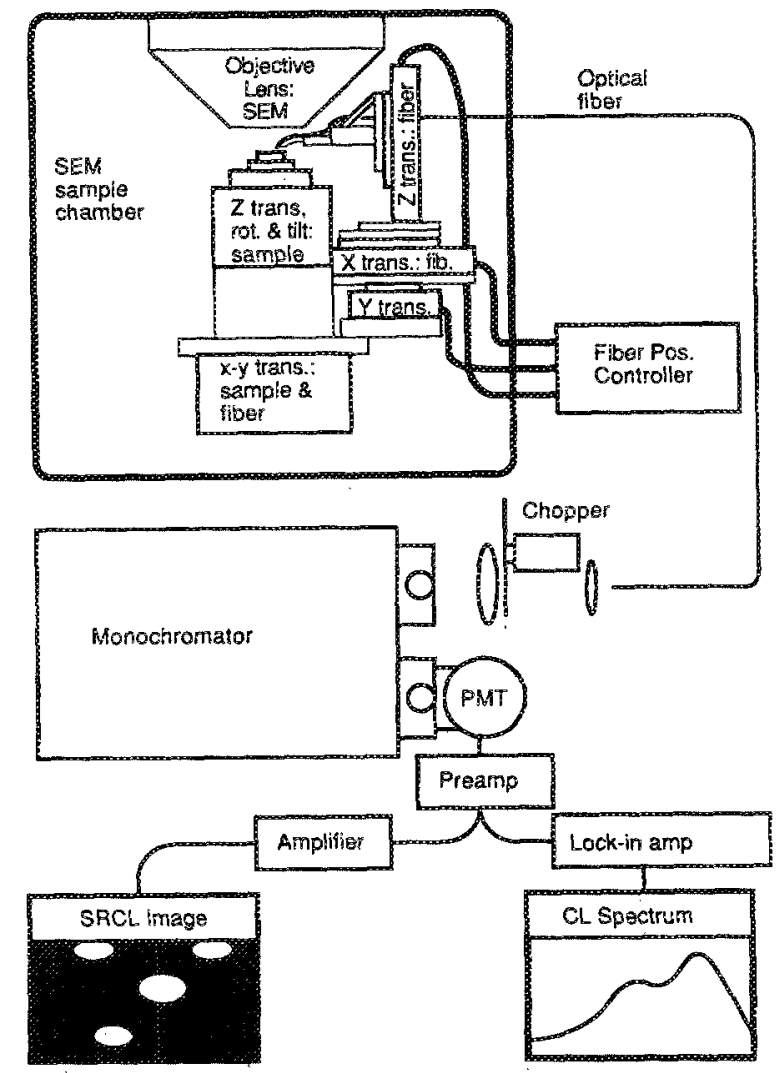

FIG. 2. Schematic diagram of the experimental apparatus. The box at the top of the figure shows the optical fiber cathodoluminescence collection system as it appears inside the scanning electron microscope chamber (compare with Fig. 1). The lower lalf of the diagram is a schematic representation of the apparatus for producing cathodoluminescence images and spectra. See text.

\section{COLLECTION OF THE CATHODOLUMINESCENCE SIGNAL}

For many applications of importance, the available cathodoluminescence signal is quite weak. For this reason, a CL light collection system must be as efficient as possible. The two important elements that determine the collection efficiency are the solid angle subtended by the collection apparatus and the efficiency with which light is guided to the detector.

In work reported to date, light collection systems for cathodoluminescence with the scanning electron microscope $^{3-6}$ and the scanning transmission electron microscope $^{7-4}$ have used collection optics with concave mirrors, planar mirrors, lenses, or some combination of these three elements. Concave mirrors, while achieving the goal of subtending as large a solid angle as possibie, restrict access to the sample. This restricts the capabilities of the microscope when the mirror is in place by interfering with apparatus designed to detect other signals from the sample.

Systems which include wavelength dispersive elements for spectral resolution of the CL signal (e.g., a monochromator) require that the detector be located ontside of the sample chamber. In order to guide the light out of the chamber, the collection optics either direct the light toward a window in the chamber or into a light pipe leading to the detection apparatus. The system which we have designed minimizes any restriction of access to the sample. Although the solid angle subtended by our collection system is less than can be achieved with a concave mirror, our efficiency in guiding the collected light to the detector is excellent. Comparison of our data with the literature indicates that our overall collection efficiency is comparable to the efficiency of systems utilizing a concave mirror.

\section{EXPERIMENTAL APPARATUS AND TECHNIQUES}

We have designed and built a cathodoluminescence system at Caltech for incorporation into the sample chamber of a Cambridge \$240 SEM (Fig. 2). Our system utilizes an optical fiber for direct collection of light from the surface of the sample. The fiber, a Newport FC-2UV multimode fiber optic cable mounted on a standard $23 / 4 \mathrm{in}$. flange, is small enough so that access to the sample by other detection systems is not restricted. Light from the sample enters the facet of the fiber, which points downward toward the region of interest. The core diameter of the fiber is $200 \mu \mathrm{m}$, and its numerical aperture is 0.2 . From this we calculate that the optimum fiber to sample distance is $0.5 \mathrm{~mm}$. In order to prevent charging of the fiber, a very thin layer of gold is evaporated onto the facet.

The fiber is held in place by inserting it into a metal sheath made from $0.010 \mathrm{in}$. wall stainless steel tubing, $1 / 16$ in. in diameter. The sheath is mounted onto a low profile support, which is designed to allow the sample to be as close to the objective lens of the SEM as possible. The minimum sample-objective working distance which we have attained is $9 \mathrm{~mm}$. The support can be manually adjusted in all three directions to center the fiber over the sample before the chamber is closed. The entire support assembly is mounted 
on a threc axis motorized transiation stage, so that the position of the optical fiber relative to the sample can be adjusted during operation of the CL system. Three vacuum compatible Oriel miniature motorized translators, model No. 16728 , are used for this purpose. Each is equipped with an optical encoder, allowing repeatable positioning of the fiber with submicron accuracy over a range of $0.5 \mathrm{in}$. The Oricl encoder mike controller, model No. 18011, is placed on the SEM console for convenient control of the ransiators during operation of the CL system.

The fiber guides the collected light horizontally out from under the objective lens of the SEM, and out of the chamber. The light is focused onto the entrance siit of a SPEX $0.34 \mathrm{~m}$ monochromator, with a $1200 \mathrm{groove} / \mathrm{mm}$ grating blazed at $750 \mathrm{~nm}$. The position of the grating is controlled by a SPEX $1673 \mathrm{C}$ Minidrive 2. The CL intensity at the selected wavelength is detected with a photomultiplier tube (PMT). A Hamamatsu R666 PMT, cooled to $40^{\circ} \mathrm{C}$ below ambient in a Pacific Instruments model 3461 housing, is used for light detection.

The signal from the PMT can be used to generate a spectrally resolved cathodoluminescence (SRCL) image (Fig. 2). This is accomplished by amplifying the PMT signal and connecting it to the auxiliary input of the SEM. The SEM video electronics generate the SRCL image and display it on the video monitor. All of the image processing electronics of the SEM are available for manipulation of the SRCL image. The SRCL image can be compared directly with any of the other types of images which the SEM can produce. Especial ly useful is the signal mixing function (Cambridge \$240 SEM), which allows the secondary electron image to be superimposed on the SRCL image. The noise can be reduced by slowing the scan rate of the electron beam and using a digital image store device to average the signal. SRCL micrographs can be taken with the video camera of the SEM.

By using the monochromator to scan an interesting region of the spectrum, we can measure local CL spectra (Fig. 2). An interesting point on the sample can be selected by moving a cursor to the appropriate place on the video display of either the secondary electron image or the SRCL image, and changing the raster of the electron beam from scan mode to spot mode. In this mode, the electron beam is being used as a movable probe for localized excitation of the sample. A mechanical light chopper is placed between the end of the fiber and the monochromator to modulate the signal. The modulated signal is connected to a current preamplifier, followed by a lock-in amplifier. The lock-in amplifier filters out much of the PMT noise by amplifying only that part of the signal which is modulated at the frequency of the light chopper. The output of the lock-in amplifier is sent to an HP $7090 \mathrm{~A}$ measurement plotting system. The plotter is controlled by an IBM PC AT through a GPIB interface bus. The monochromator controller is set to scan an interesting wavelength range, and the scan is initiated by the computer. Data from the lock-in are collected by the plotter and read into the memory of the computer. The data collected by the computer correspond to a local $\mathrm{CL}$ spectrum. The wavelength is calculated from the scan parameters, and the CL intensity is measured in millivolts from the lock-in amplifier. The computer is capable of plotting single or multiple spectra from its memory, using the HP 7090A plotter as an output device.

\section{PESULTS}

The performance of this system was demonstrated by a study of the cathodoluminescence of oval defects in $\mathrm{MBE}$ grown GaAs/AlGaAs epilayers. ${ }^{2}$ The most common macroscopic defects in the growth of $I I-V$ semiconductors by MBE are oval defects. On a (100) wafer, they are oriented with their long axis parallel to the $[01 \overline{1}]$ direction. The shape of an oval defect is probably due to a tendency of facets to propagate away from raised disturbances in the [011] direction and to propagate nearly vertically in the [011] direction. ${ }^{10}$

Oval defects in MBE grown GaAs/A1GaAs epilayers were studied in two samples. The first sample, MBE growth No. 775, consisted of a $0.1 \mu \mathrm{m}$ GaAs bufer layer, and a 2.3 $\mu \mathrm{m} n$-doped $\mathrm{Al}_{x} \mathrm{Ga}_{1-x}$ As layer grown on a semi-insulating GaAs substrate. SRCL images of some typical defects in this growth reveal strong luminescence from the oval defects at $8500 \AA$ relative to the surrounding area $[\mathrm{Fig} .3(\mathrm{c})]$, while the CL intensity from the oval defects is diminished at 8100 A [Fig. 3(b)]. Local CL spectra of the defect core and of

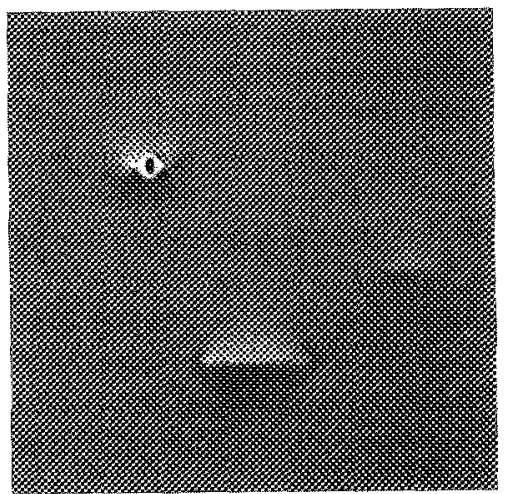

(a) $10 \mu \mathrm{m}$

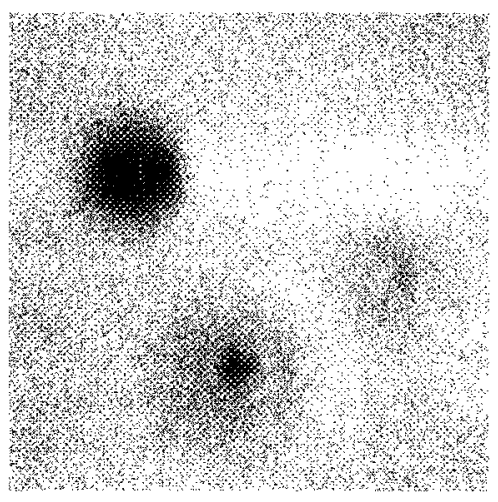

(b) $\lambda=8100 \AA$

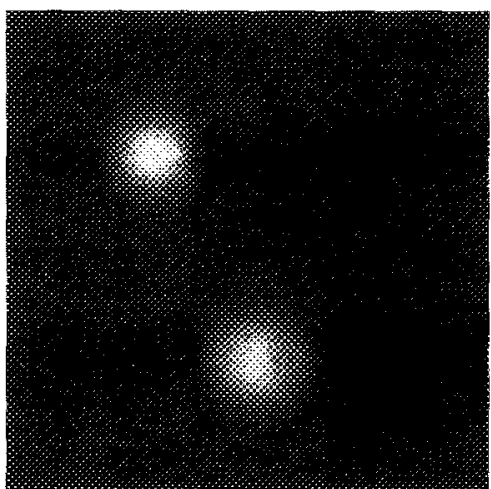

(c) $\lambda=8500 \AA$

FiG. 3. Oval defects in MBE growth No.775. (a) Secondary electron image of three typical defects; (b) spectrally resolved cathodoluminescence image (SRCI) of the same area at $8100 \AA$. showing that the cores of two of the defects do not luminesce as strongly as the rest of the sample at this wavelength; (c) SRCL image at $8500 \mathrm{~A}$, showing that the wo cefects referred to in Fig. 3(b) contain an enhanced concentration of galliwm. 


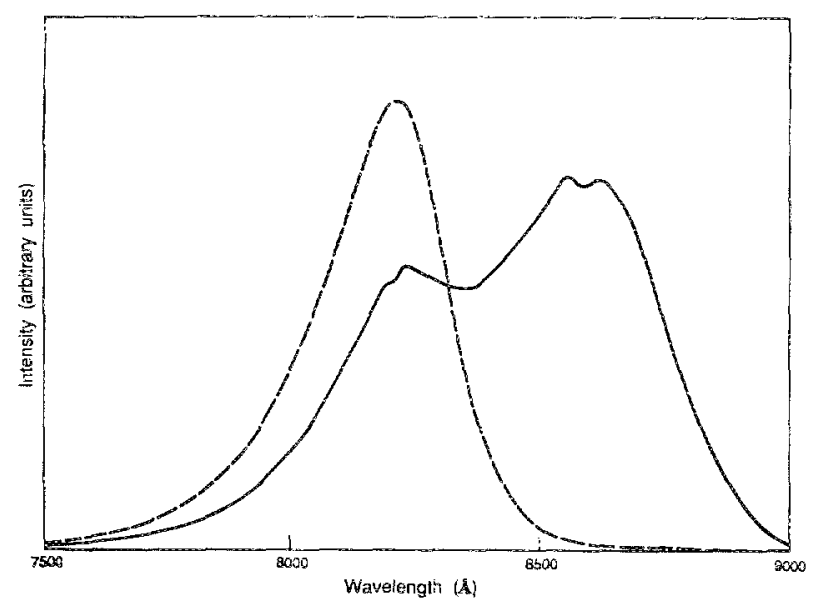

Fig. 4. Local cathodoluminescence (CL) spectra of an oval defect in MBE growth No. 775 [the upper left defect in Fig. 3(a)]. (a) Solid line: $\mathrm{CL}$ spectrum at the core of the defect; (b) broken line: $\mathrm{CL}$ spectrum from an area away from the defect. The spectra show this defect contains an enhanced concentration of gallium relative to the defect free areas of the sample.

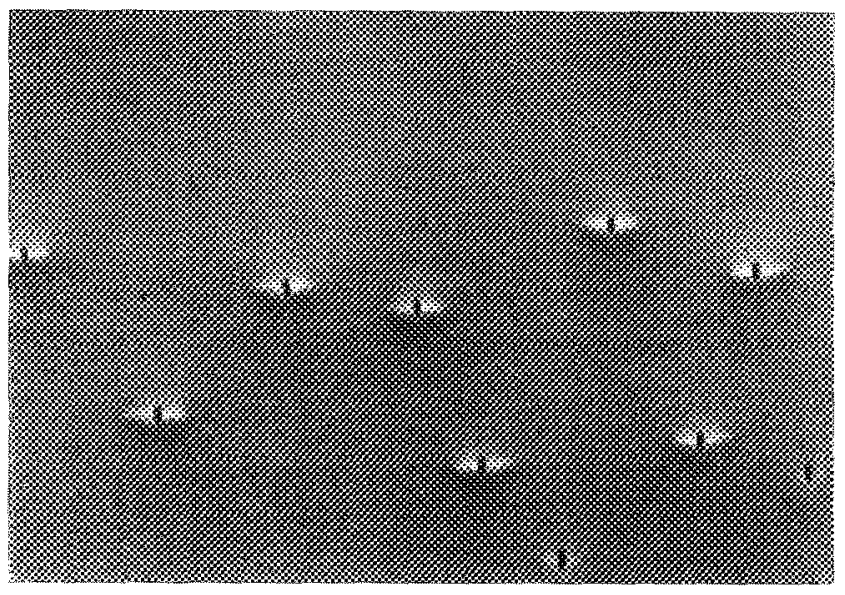

(a) 10 um

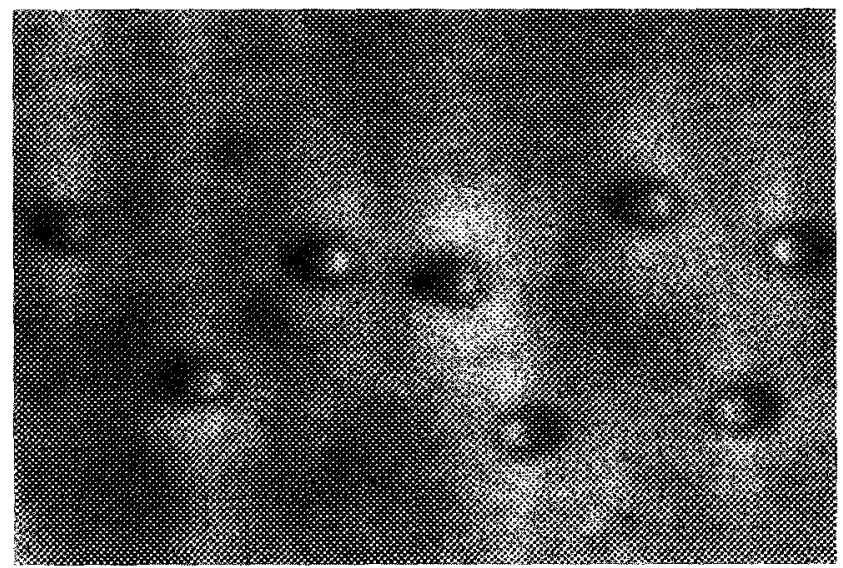

(c) $\lambda=7600 \AA$ defect-free areas of the sample show a peak at $8600 \AA$, which is present only in the vicinity of the defects (Fig. 4). The peak at $8100 \mathrm{~A}$ corresponds to a bulk aluminum fraction of 0.086 , whereas the peak at $8600 \AA$ corresponds to an aluminum fraction of 0.014 .

The second sample was a doped quantum well structure, $\mathrm{MBE}$ growth No. 562. The basic leatures of the structure are a $130 \AA$ quantum well, bounded by a $500 \AA$-doped $\mathrm{Al}_{x} \mathrm{Ga}_{1}$ As surface layer and a $0.64 \mu \mathrm{m} n$-doped $\mathrm{A} 1_{x} \mathrm{Ga}_{1-x}$ As layer. Bereath this structure is a five period superlattice $0.12 \mu \mathrm{m}$ wide and a $0.75 \mu \mathrm{m} n$-doped GaAs layer. SRCL images and local spectra of the oval defects in MBE growth No. 562 reveal a dramatic spatial and spectral dependence of the $\mathrm{CL}$ emission (Figs. 5 and 6). The bulk aluminum fraction in this sample is 0.30 , corresponding to the peak at $6900 \mathrm{~A}$ [Fig. 6 (d) ]. The aluminum fraction in the halo is 0.29 , corresponding to the peak at $6945 \mathrm{~A}$ [Figs. $5(b)$ and $6(c)]$. The aluminum fraction near the center of the defect is 0.17 , corresponding to the peak at $7600 \AA$ [Figs. $5(c)$ and $6(b)]$.

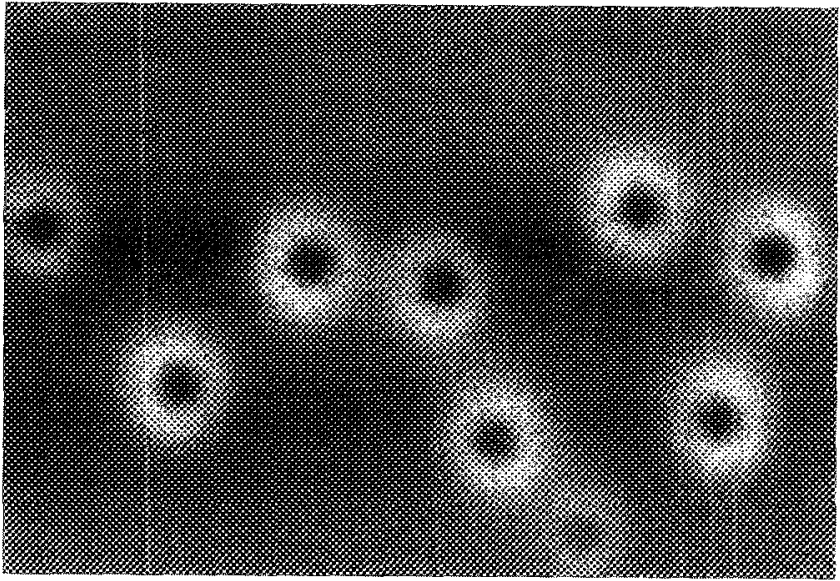

(b) $\lambda=7000 \AA$

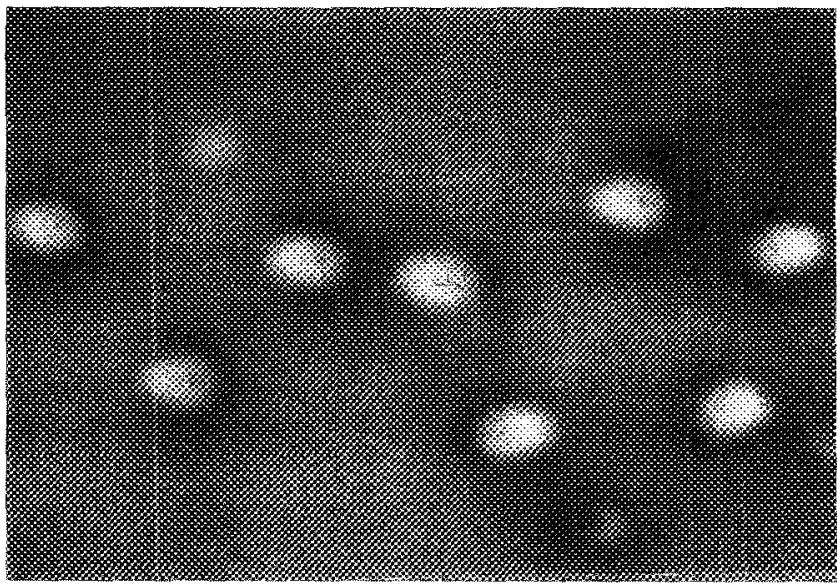

(d) $\lambda=8500 \AA$

Fra. 5. Oval defects in MBE growth No. 562. (a) Secondary electron image of a typical area of the sample; (b) spectrally resolved cathodoluminescence (SRCL) image of the same area at $7000 \AA$, showing a bright halo surrounding each of the defects. The emission from the halo corresponds to a sloped area surrounding each of the defects. The sloped area appears in optical micrographs, but they do not exhibit enough contrast to appear in secondary electron images taken by the electron microscope. (c) SRCL image at $8500 \AA$. This wavelength corresponds to emission from the quantum well. 


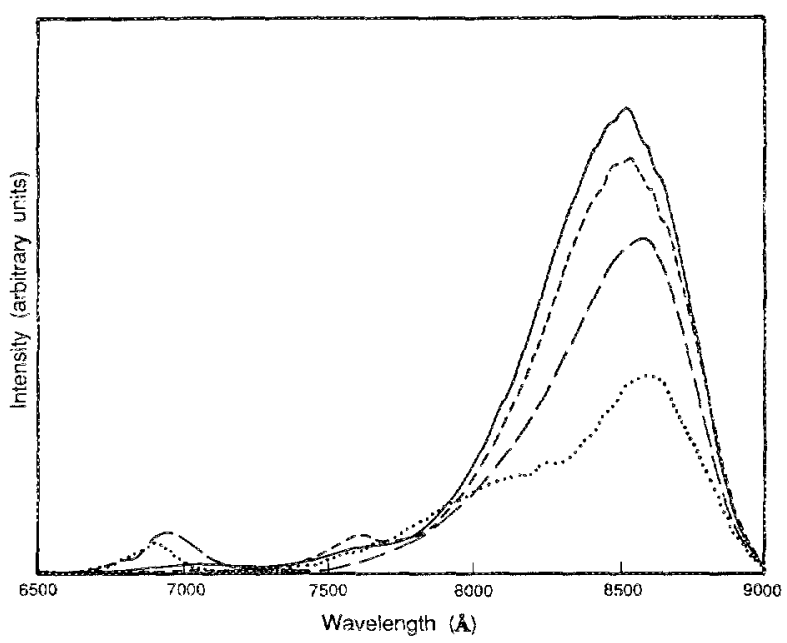

FIG. 6. Local cathodoluminescence (CL) spectra of an oval defect in MBE growth No. 562. (a) Solid line: Cl spectrum of the defect core; (b) short dashes: CL spectrum of an area centered $0.5 \% \mathrm{~m}$ from the defect core. The peak at $7600 \AA$ corresponds to an aluminum fraction of 0.17 ; (c) long dashes: CL spectrum of an area in the halo region, $2.3 \mu \mathrm{m}$ from the defect core. The peak at $6945 \AA$ corresponds to an aluminum fraction of 0.29 ; (d) dotted hine: CL spectrum away from the defects. 'The peak at $6900 \AA$ corresponds to an aluminum fraction of 0.30 .

Our observation of an enhanced concentration of gallium in the oval defect core is consistent with proposed mechanisms for the origin of defects involving localized accumulation of excess gallium during the growth. Such mechanisms include spitting of gallium from the effusion cell, nucleation of gallium droplets by a chemical reaction of $\mathrm{G} a_{2} \mathrm{O}$ formed in the $\mathrm{Ga}$ effusion cell, ${ }^{12-14}$ and gallium agglomeration around a particle on the wafer surface. ${ }^{15 \cdots 17}$

\section{ACKNOWLEDGMENTS}

The authors would like to express their appreciation to Hadis Morkoç and Lars Eng for many helpful discussions.
We would also like to thank Larry Begay for his skillful machining of the fiber mount and adapter plates.

This work was supported by the Office of Naval Research, the JPL SDIO-ISTC, and Caltech's Program in Advanced Technologies, sponsored by Aerojet General, General Motors, and TRW.

'B. G. Yacobi and D. B. Holt, J. Appl. Phys. 59, R1 (1986).

${ }^{2}$ Michael E. Hoenk and Kerry J. Vahila, Appl. Phys. Lett. 53, 2062 (1988).

${ }^{3}$ E. M. Hörl, Micron 3, 540 (1972).

4J. B. Steyn, P. Giles, and D. B. Holt, J. Microsc. 107, 107 (1976).

'S. M. Davidson and A. Rasul, J. Phys. E 10, 43 (1977).

6. Beauvineau and J. Semo, Rev. Sci. Instrum. 53, 1573 (1982).

7P. M. Petroff, D. V. Lang, J. L. Strudel, and R. A. Logan, Scanning ElecIron Microscopy/1978, edited by O. Johari (SEM, Chicago, 1978), Vol. I, p. 325 .

${ }^{*} \mathrm{~S}$. Myhajlenko, Electron Microscopy and Analysis, 1983, edited by P. Doig, Institute Physics Conference Series No. 68 (Institute of Physics, London, 1984), p. 111-114.

9 S. D. Berger and L. M. Brown, ibid., p. 115-118 (1984).

16J.S. Smith, Ph.D. thesis, California Institute of Technology, Department of Appl. Phys., 1986.

"C. E. C. Wood, L. Rathbun, H. Ohno, and D. DeSimone, J. Cryst. Growth 51, 299 (1981).

${ }^{12}$ Young G. Chai and Robert Chow, Appl. Phys. Lutt. 38, 796 (1981).

${ }^{13}$ G. D. Pettit, J. M. Wooda1, S. L. Wright, P. D. Kirchner, and J. L. Freeouf, J. Vac. Sci. Technol. B 2, 241 (1984)

${ }^{14} \mathrm{~K}$. A kimoto, M. Dohsen, M. Arai, and N. Watanabe, J. Cryst. Growth 73 , $117(1985)$.

${ }^{1.5}$ Shang-lin Weng, C. Webb, Y. G. Chai, and S. G. Bandy, Appl. Phys. Lett. 47, 391 (1985).

'S. Matteson and H. D. Shih, Appl. Phys. Lett, 48, 47 (1986).

${ }^{7}$ Shang-lin Weng, Appl. Phys. Lett. 49,345 (1986); J. Vac. Sci. Tcchnol. B 5, 725 (1987). 